\title{
Anticontrol of Hopf Bifurcation and Control of Chaos for a Finance System through Washout Filters with Time Delay
}

\author{
Huitao Zhao, Mengxia Lu, and Junmei Zuo \\ Department of Mathematics and Information Science, Zhoukou Normal University, Zhoukou, Henan 466001, China \\ Correspondence should be addressed to Huitao Zhao; taohuiz@sohu.com
}

Received 18 December 2013; Accepted 3 March 2014; Published 3 April 2014

Academic Editors: M. Han and Y. Xia

Copyright (C) 2014 Huitao Zhao et al. This is an open access article distributed under the Creative Commons Attribution License, which permits unrestricted use, distribution, and reproduction in any medium, provided the original work is properly cited.

A controlled model for a financial system through washout-filter-aided dynamical feedback control laws is developed, the problem of anticontrol of Hopf bifurcation from the steady state is studied, and the existence, stability, and direction of bifurcated periodic solutions are discussed in detail. The obtained results show that the delay on price index has great influences on the financial system, which can be applied to suppress or avoid the chaos phenomenon appearing in the financial system.

\section{Introduction}

For the last two decades, there have been growing interests in studying the complex dynamics of financial systems in both micro- and macroeconomics $[1,2]$. It is well known that the economic activity is a complex human behavior; it has many uncertainties, which is reflected in the nonlinear model for economic dynamics such as Goodwin's nonlinear accelerator model [3], forced van der Pol model on business cycle [4], the dynamic IS-LM model [5], and nonlinear dynamical model on finance system [6-9]. In these models, chaotic phenomena are common. However, in economic activities, chaos is undesired sometimes, so we want to control the chaotic orbits to a stable state or a periodic orbit. For example, in $[9,10]$, the authors showed that the chaotic behavior of a microeconomic model can be stabilized to various periodic orbits by means of time-delayed feedback control.

On the other hand, delays are ubiquitous in life, so it is in the social and economic activities. There are at least two ways that time delays emerge in the dynamics of economic variables. One is the time lag between the time economic decisions are made and the time the decisions bear fruit [11]. The other is the behavior of economic agents known as rational expectations [12]. So, it is very meaningful to investigate the effects of time delay on economic activity [10].

The aim of this paper is to investigate the dynamics of a financial system by considering the effect of washout filters with time delay. By analyzing the characteristic equation of linearization of the system, we theoretically prove that the Hopf bifurcations occur in the model with delay. Furthermore, by using the theory of functional differential equation and Hassard's method [13], we also give the conditions to determine the direction and stability of the bifurcating periodic solutions. Finally, numerical results are given to support the theoretical prediction.

The rest of the paper is organized as follows. In Section 2, we propose the controlled finance system through washout filters with delay. In Section 3, we study the local stability and Hopf bifurcation of the equilibria. In Section 4, using the normal form theory and the center manifold reduction, explicit formulae are derived to determine the direction of bifurcation and the stability and other properties of bifurcating periodic solutions. In Section 5, we will give some numerical simulations to support the theoretical prediction. In Section 6, a brief discussion is given.

\section{The Model}

In $[6,7]$, the authors have reported a dynamical model of financial system composed of four subblocks: production, money, stock, and labor force. By setting proper dimensions and choosing appropriate coordinates, the authors have offered the simplified financial model which describes the time variation of three variables: the interest rate $x$, the 
investment demand $y$, and the price index $z$. The model is represented by three-dimensional ODEs:

$$
\begin{aligned}
& \dot{x}=z+x(y-a), \\
& \dot{y}=1-b y-x^{2}, \\
& \dot{z}=-x-c z,
\end{aligned}
$$

where $a>0$ is the saving amount, $b>0$ is the cost per investment, and $c>0$ is the elasticity of demand of commercial market. This model is well studied in [6-9]; their results show that system (1) has abundant dynamical behaviors including Hopf bifurcation and chaos; however, the effect of time delay on the dynamics of this financial system was not taken into account.

In the following, we consider the effect of washout filters with time delay. We first consider a general form of dynamical system:

$$
\dot{X}=f(X ; \mu) \text {, }
$$

where $X$ is a vector and $\mu$ is a parameter. The washout-filteraided controller assumes the following structure:

$$
\begin{aligned}
& \dot{X}=f(X ; \mu)+u, \\
& \dot{w}=X_{i}-d w, \\
& u=g(\rho ; K),
\end{aligned}
$$

where $u$ is a control input, $g$ is a control function, and $d$ is the washout filter time constant. The following constraints should be fulfilled: $d>0$, which guarantees the stability of the washout filter; $g(0 ; K)=0$, which preserves the original equilibrium points.

In this paper, the controlled system is designed as follows:

$$
\begin{aligned}
& \dot{x}(t)=z(t)+x(t)(y(t)-a), \\
& \dot{y}(t)=1-b y(t)-x^{2}(t), \\
& \dot{z}(t)=-x(t)-c z(t)+u(t), \\
& \dot{u}(t)=k(z(t)-z(t-\tau))-d u(t),
\end{aligned}
$$

where $k>0$ is a control gain, $d>0$ is an accommodation coefficient, and $\tau>0$ is time delay. $u$ is the control input; differing from the time-delayed feedback controller (DFC) [10], the changing rate of controller $u$ is influenced by the time delay feedback on price index $z$ and adjusted by $u$. This system has the similar character with washout filter controller, $d>$ 0 , which guarantees the stability of the controller, and the original equilibrium points were preserved $[14,15]$.

\section{Existence of Hopf Bifurcation}

In this section, we choose the gain $k$ as a constant and investigate the effect of time delay $\tau$ on the dynamic behavior of the controlled system (4). First, the following conclusions for the uncontrolled system (1) are needed.
Lemma 1. When $c-b-a b c \leqslant 0$, that is, $1+a c-(c / b) \geqslant 0$, system (1) has a unique equilibrium $P_{0}(0,1 / b, 0)$.

Lemma 2. When $c-b-a b c>0$, that is, $1+a c-$ $(c / b)<0$, system (1) has three equilibria $P_{0}(0,1 / b, 0)$ and $P_{ \pm}( \pm \sqrt{(c-b-a b c) / c},(1+a c) / c, \mp(1 / c) \sqrt{(c-b-a b c) / c})$.

The characteristic equation of the Jacobian matrix at the equilibria $P_{ \pm}$of system (1) is

$$
\lambda^{3}+a_{1} \lambda^{2}-a_{2} \lambda+a_{3}=0
$$

where $a_{1}=\left(c^{2}+b c-1\right) / c, a_{2}=\left(-b c^{2}-2 c+3 b+2 a b c\right) / c$, and $a_{3}=-2 b+2 c-2 a b c$. Then, from Routh-Hurwitz criterion, the real parts of all the roots of the above equation are negative if and only if the conditions

(H1) $a_{1}>0, a_{3}>0$, and $a_{1} a_{2}-a_{3}>0$ hold.

\section{Lemma 3.}

(i) The equilibrium $P_{0}(0,1 / b, 0)$ of system (1) is stable when $1+a c-(c / b)>0$ and $c+a-(1 / b)>0$.

(ii) The equilibria $P_{ \pm}$of system (1) are stable when $c-b-$ $a b c>0$ and (H1) hold.

3.1. Hopf Bifurcation from the Stable Equilibrium $P_{0}$. The linear equation of the controlled system (4) at $P_{0}$ (where $u_{0}=$ $0)$ is

$$
\begin{aligned}
& \dot{x}(t)=z(t)-\left(a-\frac{1}{b}\right) x(t), \\
& \dot{y}(t)=-b y(t), \\
& \dot{z}(t)=-x(t)-c z(t)+u(t), \\
& \dot{u}(t)=k(z(t)-z(t-\tau))-d u(t) .
\end{aligned}
$$

The associated characteristic equation of the linearized system is

$$
\operatorname{det}\left(\begin{array}{cccc}
\lambda+a-\frac{1}{b} & 0 & -1 & 0 \\
0 & \lambda+b & 0 & 0 \\
1 & 0 & \lambda+c & -1 \\
0 & 0 & k\left(e^{-\lambda \tau}-1\right) & \lambda+d
\end{array}\right)=0 .
$$

That is,

$$
(\lambda+b)\left[\lambda^{3}+p_{1} \lambda^{2}+p_{2} \lambda+p_{3}+\left(q_{1} \lambda+q_{2}\right) e^{-\lambda \tau}\right]=0,
$$

where

$$
\begin{aligned}
& p_{1}=a+c+d-\frac{1}{b} \\
& p_{2}=\left(a-\frac{1}{b}\right)(c+d)+c d-k+1, \\
& p_{3}=\left(a-\frac{1}{b}\right)(c d-k)+d \\
& q_{1}=k, \quad q_{2}=k\left(a-\frac{1}{b}\right) .
\end{aligned}
$$


It is well known that the equilibrium $P_{0}(0,1 / b, 0,0)$ is stable if all the roots of (8) have negative real parts. Obviously, (8) always has a negative root $\lambda=-b$, for all $\tau \geqslant 0$, so, we only need to investigate the third transcendental polynomial equation:

$$
\lambda^{3}+p_{1} \lambda^{2}+p_{2} \lambda+p_{3}+\left(q_{1} \lambda+q_{2}\right) e^{-\lambda \tau}=0
$$

Obviously, if $\lambda= \pm i \omega(\omega>0)$ is a pair of pure imaginary roots of (8), then $\omega$ satisfies

$$
\begin{aligned}
-i \omega^{3} & -p_{1} \omega^{2}+i p_{2} \omega+p_{3} \\
& +\left(i q_{1} \omega+q_{2}\right)(\cos \omega \tau-i \sin \omega \tau)=0 .
\end{aligned}
$$

Separating the real and imaginary parts, we have

$$
\begin{aligned}
& \omega^{3}-p_{2} \omega=q_{1} \omega \cos \omega \tau-q_{2} \sin \omega \tau, \\
& p_{1} \omega^{2}-p_{3}=q_{1} \omega \sin \omega \tau+q_{2} \cos \omega \tau,
\end{aligned}
$$

and it follows that

$$
\omega^{6}+p \omega^{4}+q \omega^{2}+r=0
$$

where

$$
\begin{aligned}
& p=p_{1}^{2}-2 p_{2}, \\
& q=p_{2}^{2}-2 p_{1} p_{3}-q_{1}^{2}, \\
& r=p_{3}^{2}-q_{2}^{2} .
\end{aligned}
$$

Let $\zeta=\omega^{2}$, and (13) becomes

$$
\zeta^{3}+p \zeta^{2}+q \zeta+r=0
$$

Denote $h(\zeta)=\zeta^{3}+p \zeta^{2}+q \zeta+r$. We have the following.

Lemma 4. For (15), one has the following results:

(i) if $r<0$, then (15) has at least one positive root;

(ii) if $r \geqslant 0$ and $\Delta=p^{2}-3 q \leqslant 0$, then (15) has no positive root;

(iii) if $r \geqslant 0$ and $\Delta=p^{2}-3 q>0$, then (15) has positive root if and only if $\zeta_{1}=(1 / 3)(-p+\sqrt{\Delta})>0$ and $h\left(\zeta_{1}\right) \leqslant 0$.

Without loss of generality, suppose that $\zeta_{i}(i=1,2,3)$ are positive roots of (15). Then, $\omega_{i}=\sqrt{\zeta_{i}}$ is a root of (13). From (12), we have

$$
\begin{gathered}
\tau_{i}^{j} \\
=\frac{1}{\omega_{i}}\left\{\arccos \frac{q_{1} \omega^{4}+\left(p_{1} q_{2}-p_{2} q_{1}\right) \omega^{2}-q_{2} p_{3}}{q_{1}^{2} \omega^{2}+q_{2}^{2}}+2 j \pi\right\}, \\
j=0,1,2, \ldots
\end{gathered}
$$

Denote

$$
\tau_{0}=\min \left\{\tau_{i}^{0} \mid i=1,2,3\right\}
$$

Substituting $\lambda(\tau)$ into (10) and taking the derivative with respect to $\tau$, we have

$$
\left[\frac{d \lambda}{d \tau}\right]^{-1}=\frac{\left(3 \lambda^{2}+2 p_{1} \lambda+p_{2}\right) e^{\lambda \tau}+q_{1}}{\lambda\left(q_{1} \lambda+q_{2}\right)}-\frac{\tau}{\lambda} .
$$

From (12) and (18), through tedious computing, we get

$$
\left[\frac{d \lambda}{d \tau}\right]_{\tau=\tau_{i}^{j}}^{-1}=\frac{\zeta_{i}}{\Lambda} h^{\prime}\left(\zeta_{i}\right)
$$

where $\Lambda=\omega_{i}^{2}\left(q_{1} \omega_{i}^{2}+q_{2}\right)$. Since $\zeta_{i}>0$, then $[d \lambda / d \tau]_{\tau=\tau_{i}^{j}}^{-1}$ and $h^{\prime}\left(\zeta_{i}\right)$ have the same sign. Thus, from Lemmas 3 and 4 , we have the following theorem.

Theorem 5. Suppose $1+a c-(c / b)>0$ and $c+a-(1 / b)>0$; then, one has the following:

(i) if $r \geqslant 0$ and $\Delta=p^{2}-3 q \leqslant 0$, then the equilibrium $P_{0}$ is stable for all $\tau \geqslant 0$;

(ii) if $r<0$ (or $r \geqslant 0$ and $\Delta=p^{2}-3 q>0$ ) and $h^{\prime}\left(\zeta_{i}\right) \neq 0$, then, when $\tau \in\left[0, \tau_{0}\right)$, the equilibrium $P_{0}$ is stable, and system (4) undergoes Hopf bifurcation at $P_{0}$ when $\tau$ passes through $\tau_{0}$.

3.2. Hopf Bifurcation from the Stable Equilibria $P_{ \pm}$. In this subsection, we assume that system (1) has two stable equilibria $P_{ \pm}( \pm \sqrt{(c-b-a b c) / c},(1+a c) / c, \mp(1 / c) \sqrt{(c-b-a b c) / c})$. Due to the symmetry of $P_{+}$and $P_{-}$, it is sufficient to analyze the stability of $P_{+}$.

Let $P_{+}=(\tilde{x}, \tilde{y}, \tilde{y})=(\sqrt{(c-b-a b c) / c},(1+a c) / c$, $-(1 / c) \sqrt{(c-b-a b c) / c})$. By the linear transforms $(t)=x(t)-$ $\tilde{x}, Y(t)=y(t)-\tilde{y}, Z(t)=z(t)-\tilde{z}$, and $U(t)=u(t)$, the linear equation of the controlled system (4) at $P_{+}$is

$$
\begin{aligned}
& \dot{X}(t)=Z(t)-(a-\tilde{y}) X(t)+\tilde{x} Y(t), \\
& \dot{Y}(t)=-b Y(t)-2 \tilde{x} X(t) \\
& \dot{Z}(t)=-X(t)-c Z(t)+U(t), \\
& \dot{U}(t)=k(Z(t)-Z(t-\tau))-d U(t) .
\end{aligned}
$$

The associated characteristic equation of system (20) is

$$
\operatorname{det}\left(\begin{array}{cccc}
\lambda+a-\tilde{y} & -\tilde{x} & -1 & 0 \\
2 \tilde{x} & \lambda+b & 0 & 0 \\
1 & 0 & \lambda+c & -1 \\
0 & 0 & k\left(e^{-\lambda \tau}-1\right) & \lambda+d
\end{array}\right)=0
$$

Expand (21), and we have

$$
\lambda^{4}+\rho_{1} \lambda^{3}+\rho_{2} \lambda^{2}+\rho_{3} \lambda+\rho_{4}+\left(\sigma_{1} \lambda^{2}+\sigma_{2} \lambda+\sigma_{3}\right) e^{-\lambda \tau}=0
$$


where

$$
\begin{aligned}
& \rho_{1}=a+b+c+d-\tilde{y}, \\
& \rho_{2}=b(a-\tilde{y})+2 \tilde{x}^{2}+c d-k+(c+b-\tilde{y})(c+d), \\
& \rho_{3}=(a+c-\tilde{y})(c d-k)+(c+d)\left(a b-b \tilde{y}+2 \tilde{x}^{2}\right), \\
& \rho_{4}=\left(a b-b \tilde{y}+2 \tilde{x}^{2}\right)(c d-k), \\
& \sigma_{1}=k, \quad \sigma_{2}=k(a+b-\tilde{y}), \\
& \sigma_{3}=k\left(a b-b \tilde{y}+2 \tilde{x}^{2}\right) .
\end{aligned}
$$

Suppose $i \omega$ is a root of (22); then, $\omega$ satisfies

$$
\begin{aligned}
\omega^{4}-\rho_{2} \omega^{2}+\rho_{4} & =\left(\sigma_{1} \omega^{2}-\sigma_{3}\right) \cos \omega \tau-\sigma_{2} \omega \sin \omega \tau, \\
\rho_{1} \omega^{3}-\rho_{3} \omega & =\sigma_{2} \omega \cos \omega \tau+\left(\sigma_{1} \omega^{2}-\sigma_{3}\right) \sin \omega \tau,
\end{aligned}
$$

which lead to

$$
\omega^{8}+\kappa_{1} \omega^{6}+\kappa_{2} \omega^{4}+\kappa_{3} \omega^{2}+\kappa_{4}=0,
$$

where

$$
\begin{aligned}
& \kappa_{1}=\rho_{1}^{2}-2 \rho_{2}, \quad \kappa_{2}=\rho_{2}^{2}+2 \rho_{4}-2 \rho_{1} \rho_{3}-\sigma_{1}^{2}, \\
& \kappa_{3}=\rho_{3}^{2}-2 \rho_{2} \rho_{4}+2 \sigma_{1} \sigma_{3}-\sigma_{2}^{2}, \quad \kappa_{4}=\rho_{4}^{2}-\sigma_{3}^{2} .
\end{aligned}
$$

Denote $z=\omega^{2}$; then, (24) becomes

$$
z^{4}+\kappa_{1} z^{3}+\kappa_{2} z^{2}+\kappa_{3} z+\kappa_{4}=0
$$

Denote

$$
g(z)=z^{4}+\kappa_{1} z^{3}+\kappa_{2} z^{2}+\kappa_{3} z+\kappa_{4}
$$

Clearly, if $\kappa_{4}<0$, then (27) has at least one positive root. Suppose $z_{k}$ is a positive root of (27); then, $\omega_{k}=\sqrt{z_{k}}$ is a root of (25). From (24), we have

$$
\begin{gathered}
\tau_{k}^{(j)}=\frac{1}{\omega_{k}}\{\arccos ( \\
\quad \sigma_{1} \omega_{k}^{6}-\left(\rho_{2} \sigma_{1}-\rho_{1} \sigma_{2}+\sigma_{3}\right) \omega_{k}^{4} \\
\left.+\left(\rho_{4} \sigma_{1}+\rho_{2} \sigma_{3}-\rho_{3} \sigma_{2}\right) \omega_{k}^{2}-\rho_{4} \sigma_{3}\right) \\
\left.\times\left(\left(\sigma_{1} \omega_{k}^{2}-\sigma_{3}\right)^{2}+\sigma_{2}^{2} \omega_{k}^{2}\right)^{-1}\right) \\
+2 j \pi\}, \quad j=0,1,2, \ldots
\end{gathered}
$$

Substituting $\lambda(\tau)$ into (22) and taking the derivative with respect to $\tau$, we obtain

$$
\begin{aligned}
& {\left[\frac{d \lambda}{d \tau}\right]^{-1}} \\
& =\frac{\left(4 \lambda^{3}+3 \rho_{1} \lambda^{2}+2 \rho_{2} \lambda+\rho_{3}\right) e^{\lambda \tau}+2 \sigma_{1} \lambda+\sigma_{2}}{\lambda\left(\sigma_{1} \lambda^{2}+\sigma_{2} \lambda+\sigma_{3}\right)} \\
& \quad-\frac{\tau}{\lambda} .
\end{aligned}
$$

From (24) and (30), we have

$$
\left[\frac{d \lambda}{d \tau}\right]_{\tau=\tau_{k}^{j}}^{-1}=\frac{z_{k}}{\Gamma} g^{\prime}\left(z_{k}\right),
$$

where $\Gamma=\omega_{k}^{2}\left(\left(\sigma_{3}-\sigma_{1} \omega_{k}^{2}\right)^{2}+\sigma_{2}^{2} \omega_{k}^{2}\right)$. Thus, from the above analysis, we have the following.

Theorem 6. Suppose $\kappa_{4}<0$ and $g^{\prime}\left(z_{k}\right) \neq 0$; then, system (4) undergoes Hopf bifurcation at the steady state $P_{ \pm}$when $\tau$ passes through $\tau_{k}^{(j)}$.

\section{Direction and Stability of the Hopf Bifurcation}

In Section 3, we obtain the conditions under which a family of periodic solutions bifurcate from the steady state at the critical value of $\tau$. In this section, following the ideal of [13], we derive the explicit formulae for determining the properties of the Hopf bifurcation at the critical value of $\tau$ using the normal form and the center manifold theory.

In this section, we always assume that system (4) undergoes Hopf bifurcation at the steady state $\left(x^{*}, y^{*}, z^{*}\right)$ for $\tau=$ $\tau_{i}$, and then $\pm i \omega_{i}$ is the corresponding purely imaginary roots of the characteristic equation at the steady state $\left(x^{*}, y^{*}, z^{*}\right)$.

Let $u_{1}=x-x^{*}, u_{2}=y-y^{*}, u_{3}=z-z^{*}, u_{4}=u$, $\overline{u_{i}}=u_{i}(\tau t)$, and $\tau=\tau_{i}+\mu$ and drop the bars for simplification of notations. Then, system (4) can be rewritten as a functional differential equation in $\mathbb{C}\left([-1,0], \mathbb{R}^{4}\right)$ :

$$
\dot{u}(t)=L_{\mu}\left(u_{t}\right)+f\left(\mu, u_{t}\right) \text {, }
$$

where $u=\left(u_{1}, u_{2}, u_{3}, u_{4}\right)^{T}$. For $\phi=\left(\phi_{1}, \phi_{2}, \phi_{3}, \phi_{4}\right)^{T} \in$ $\mathbb{C}\left([-1,0], \mathbb{R}^{4}\right)$,

$$
\begin{gathered}
L_{\mu}(\phi)=\left(\tau_{i}+\mu\right)\left[\begin{array}{cccc}
-a+y^{*} & x^{*} & 1 & 0 \\
2 x^{*} & -b & 0 & 0 \\
-1 & 0 & -c & 1 \\
0 & 0 & k & -d
\end{array}\right]\left[\begin{array}{l}
\phi_{1}(0) \\
\phi_{2}(0) \\
\phi_{3}(0) \\
\phi_{4}(0)
\end{array}\right] \\
+\left(\tau_{i}+\mu\right)\left[\begin{array}{cccc}
0 & 0 & 0 & 0 \\
0 & 0 & 0 & 0 \\
0 & 0 & 0 & 0 \\
0 & 0 & -k & 0
\end{array}\right]\left[\begin{array}{l}
\phi_{1}(-1) \\
\phi_{2}(-1) \\
\phi_{3}(-1) \\
\phi_{4}(-1)
\end{array}\right], \\
f(\mu, \phi)=\left(\tau_{i}+\mu\right)\left[\begin{array}{c}
\phi_{1}(0) \phi_{2}(0) \\
\phi_{1}^{2}(0) \\
0 \\
0
\end{array}\right] .
\end{gathered}
$$

Obviously, $L(\mu)$ is a continuous linear function mapping $\mathbb{C}\left([-1,0], \mathbb{R}^{4}\right)$ into $\mathbb{R}^{4}$. By the Riesz representation theorem, there exists a $4 \times 4$ matrix function $\eta(\theta, \mu)(-1 \leqslant \theta \leqslant 0)$, whose elements are of bounded variation such that

$$
L_{\mu} \phi=\int_{-1}^{0} d \eta(\theta, 0) \phi(\theta), \quad \text { for } \phi \in \mathbb{C}\left([-1,0], \mathbb{R}^{4}\right) \text {. }
$$


In fact, we can choose

$$
\begin{aligned}
\eta(\theta, \mu)= & \left(\tau_{i}+\mu\right)\left[\begin{array}{cccc}
-a+y^{*} & x^{*} & 1 & 0 \\
2 x^{*} & -b & 0 & 0 \\
-1 & 0 & -c & 1 \\
0 & 0 & k & -d
\end{array}\right] \delta(\theta) \\
& +\left(\tau_{i}+\mu\right)\left[\begin{array}{cccc}
0 & 0 & 0 & 0 \\
0 & 0 & 0 & 0 \\
0 & 0 & 0 & 0 \\
0 & 0 & -k & 0
\end{array}\right] \delta(\theta+1),
\end{aligned}
$$

where $\delta$ denote Dirac-delta function. For $\phi \in \mathbb{C}\left([-1,0], \mathbb{R}^{4}\right)$, define

$$
\begin{aligned}
& A(\mu) \phi(\theta)= \begin{cases}\frac{d \phi(\theta)}{d \theta}, & \theta \in[-1,0), \\
\int_{-1}^{0} d \eta(s, \mu) \phi(s), & \theta=0,\end{cases} \\
& R(\mu) \phi(\theta)= \begin{cases}0, & \theta \in[-1,0), \\
f(\mu, \phi), & \theta=0 .\end{cases}
\end{aligned}
$$

Then, when $\theta=0$, the system

$$
\dot{u}_{t}=A(\mu) u_{t}+R(\mu) u_{t}
$$

is equivalent to the system (32), where $u_{t}(\theta)=u(t+\theta)$ and $\theta \in[-1,0]$. For $\psi \in \mathbb{C}^{1}\left([0,1],\left(\mathbb{R}^{4}\right)^{*}\right)$, define

$$
A^{*} \psi(s)= \begin{cases}-\frac{d \psi(s)}{d s}, & s \in(0,1], \\ \int_{-1}^{0} d \eta^{T}(t, 0) \psi(-t), & s=0,\end{cases}
$$

and a bilinear inner product

$$
\begin{aligned}
\langle\psi(s), \phi(\theta)\rangle= & \bar{\psi}(0) \phi(0) \\
& -\int_{-1}^{0} \int_{\xi=0}^{\theta} \bar{\psi}(\xi-\theta) d \eta(\theta) \phi(\xi) d \xi,
\end{aligned}
$$

where $\eta(\theta)=\eta(\theta, 0)$; let $A=A(0)$; then, $A$ and $A^{*}$ are adjoint operators. By the discussion in Section 3, we know that $\pm i \omega_{i} \tau_{i}$ are eigenvalues of $A$. Thus, they are also eigenvalues of $A^{*}$. We first need to compute the eigenvector of $A$ and $A^{*}$ corresponding to $i \omega_{i} \tau_{i}$ and $-i \omega_{i} \tau_{i}$, respectively.

Suppose that $q(\theta)=(1, \alpha, \beta, \gamma)^{T} e^{i \omega_{i} \tau_{i} \theta}$ is the eigenvector of $A$ corresponding to $i \omega_{i} \tau_{i}$. Then, $A q(\theta)=i \omega_{i} \tau_{i} q(\theta)$. It follows from the definition of $A, L_{\mu} \phi$, and $\eta(\theta, \mu)$ that

$$
\begin{gathered}
\tau_{i}\left[\begin{array}{cccc}
i \omega_{i}+a-y^{*} & -x^{*} & -1 & 0 \\
-2 x^{*} & i \omega_{i}+b & 0 & 0 \\
1 & 0 & i \omega_{i}+c & -1 \\
0 & 0 & -k+k e^{-i \omega_{i} \tau_{i}} & i \omega_{i}+d
\end{array}\right] q(0) \\
\quad=\left[\begin{array}{l}
0 \\
0 \\
0 \\
0
\end{array}\right]
\end{gathered}
$$

Thus, we can easily obtain $\alpha=2 x^{*} /\left(i \omega_{i}+b\right), \beta=i \omega_{i}+a-$ $y^{*}-\alpha x^{*}, \gamma=1+\left(i \omega_{i}+c\right) \beta$, and $q(0)=(1, \alpha, \beta, \gamma)^{T}$.

Similarly, let $q^{*}(s)=D\left(1, \alpha^{*}, \beta^{*}, \gamma^{*}\right) e^{i \omega_{i} \tau_{i} s}$ be the eigenvector of $A^{*}$ corresponding to $-i \omega_{i} \tau_{i}$. By the definition of $A^{*}$, we can compute $\alpha^{*}=-x^{*} /\left(i \omega_{i}-b\right), \beta^{*}=i \omega_{i}-a+y^{*}+2 x^{*} \alpha^{*}$, and $\gamma^{*}=-\left(\beta^{*} /\left(i \omega_{i}-d\right)\right)$.

In order to assure that $\left\langle q^{*}(s), q(\theta)\right\rangle=1$, we need to determine the value of $D$. From (39), we have

$$
\begin{aligned}
& \left\langle q^{*}(s), q(\theta)\right\rangle \\
& =\bar{D}\left(1, \overline{\alpha^{*}}, \overline{\beta^{*}}, \overline{\gamma^{*}}\right)(1, \alpha, \beta, \gamma)^{T} \\
& \quad-\int_{-1}^{0} \int_{\xi=0}^{\theta} \bar{D}\left(1, \overline{\alpha^{*}}, \overline{\beta^{*}}, \overline{\gamma^{*}}\right) e^{-i \omega_{i} \tau_{i}(\xi-\theta)} d \eta(\theta) \\
& \quad \times(1, \alpha, \beta, \gamma)^{T} e^{i \omega_{i} \tau_{i} \xi} d \xi \\
& =\bar{D}\left\{1+\alpha \overline{\alpha^{*}}+\beta \overline{\beta^{*}}+\gamma \overline{\gamma^{*}}\right. \\
& =\bar{D}\left\{1+\alpha \overline{\alpha^{*}}+\beta \overline{\beta^{*}}+\gamma \overline{\gamma^{*}}-k \beta \overline{\gamma^{*}} e^{-i \omega_{i} \tau_{i}}\right\} .
\end{aligned}
$$

Thus, we can choose

$$
\bar{D}=\left\{1+\alpha \overline{\alpha^{*}}+\beta \overline{\beta^{*}}+\gamma \overline{\gamma^{*}}-k \beta \overline{\gamma^{*}} e^{-i \omega_{i} \tau_{i}}\right\}^{-1},
$$

such that $\left\langle q^{*}(s), q(\theta)\right\rangle=1,\left\langle q^{*}(s), \bar{q}(\theta)\right\rangle=0$.

In the following, we first compute the coordinates to describe the center manifold $C_{0}$ at $\mu=0$. Define

$$
z(t)=\left\langle q^{*}, u_{t}\right\rangle, \quad W(t, \theta)=u_{t}(\theta)-2 \operatorname{Re}\{z(t) q(\theta)\} .
$$

On the center manifold $C_{0}$, we have

$$
\begin{aligned}
W(t, \theta)= & W(z(t), \bar{z}(t), \theta) \\
= & W_{20}(\theta) \frac{z^{2}}{2}+W_{11}(\theta) z \bar{z}+W_{02}(\theta) \frac{\bar{z}^{2}}{2} \\
& +W_{30}(\theta) \frac{z^{3}}{6}+\cdots,
\end{aligned}
$$

where $z$ and $\bar{z}$ are local coordinates for center manifold $C_{0}$ in the direction of $q$ and $\bar{q}$. Note that $W$ is real if $u_{t}$ is real. We consider only real solutions. For the solution $u_{t} \in C_{0}$, since $\mu=0$, we have

$$
\begin{aligned}
\dot{z}= & i \omega_{i} \tau_{i} z \\
& +\left\langle q^{*}(\theta), f(0, W(z(t), \bar{z}(t), \theta)+2 \operatorname{Re}\{z(t) q(\theta)\})\right\rangle \\
= & i \omega_{i} \tau_{i} z \\
& +\overline{q^{*}}(0) f(0, W(z(t), \bar{z}(t), 0)+2 \operatorname{Re}\{z(t) q(0)\}) \\
\triangleq & i \omega_{i} \tau_{i} z+\overline{q^{*}}(0) f_{0}(z, \bar{z})=i \omega_{i} \tau_{i} z+g(z, \bar{z}),
\end{aligned}
$$


where

$$
\begin{aligned}
g(z, \bar{z})= & \overline{q^{*}}(0) f_{0}(z, \bar{z}) \\
= & g_{20}(\theta) \frac{z^{2}}{2}+g_{11}(\theta) z \bar{z}+g_{02}(\theta) \frac{\bar{z}^{2}}{2} \\
& +g_{21}(\theta) \frac{z^{2} \bar{z}}{2}+\cdots .
\end{aligned}
$$

From (43) and (44), we have

$$
\begin{aligned}
u_{t}(\theta) & =\left(u_{1 t}(\theta), u_{2 t}(\theta), u_{3 t}(\theta), u_{4 t}(\theta)\right)^{T} \\
& =W(t, \theta)+z q(\theta)+\bar{z} \bar{q}(\theta) .
\end{aligned}
$$

In addition, $q(\theta)=(1, \alpha, \beta, \gamma)^{T} e^{i \omega_{i} \tau_{i} \theta}$; then,

$$
\begin{aligned}
u_{1 t}(0)= & +\bar{z}+W_{20}^{(1)}(0) \frac{z^{2}}{2}+W_{11}^{(1)}(0) z \bar{z} \\
& +W_{02}^{(1)}(0) \frac{\bar{z}^{2}}{2}+O\left(|(z, \bar{z})|^{3}\right), \\
u_{2 t}(0)= & \alpha z+\bar{\alpha} \bar{z}+W_{20}^{(2)}(0) \frac{z^{2}}{2} \\
& +W_{11}^{(2)}(0) z \bar{z}+W_{02}^{(2)}(0) \frac{\bar{z}^{2}}{2}+O\left(|(z, \bar{z})|^{3}\right), \\
u_{3 t}(0)= & \beta z+\bar{\beta} \bar{z}+W_{20}^{(3)}(0) \frac{z^{2}}{2} \\
& +W_{11}^{(3)}(0) z \bar{z}+W_{02}^{(3)}(0) \frac{\bar{z}^{2}}{2}+O\left(|(z, \bar{z})|^{3}\right), \\
u_{4 t}(0)= & \gamma z+\bar{\gamma} \bar{z}+W_{20}^{(4)}(0) \frac{z^{2}}{2}+W_{11}^{(4)}(0) z \bar{z} \\
& +W_{02}^{(4)}(0) \frac{\bar{z}^{2}}{2}+O\left(|(z, \bar{z})|^{3}\right) .
\end{aligned}
$$

By the definition of $f\left(\mu, x_{t}\right)$, we have

$$
g(z, \bar{z})=\bar{D} \tau_{i}\left(1, \overline{\alpha^{*}}, \overline{\beta^{*}}, \overline{\gamma^{*}}\right)\left[\begin{array}{c}
u_{1 t}(0) u_{2 t}(0) \\
u_{1 t}^{2}(0) \\
0 \\
0
\end{array}\right] .
$$

Substituting $u_{1 t}(0), u_{2 t}(0), u_{3 t}(0)$, and $u_{4 t}(0)$ into the above equation and comparing the coefficients with (46), we get

$$
\begin{aligned}
g_{20} & =2 \bar{D} \tau_{i}\left(\alpha+\overline{\alpha^{*}}\right), \\
g_{11} & =\bar{D} \tau_{i}\left(\alpha+\bar{\alpha}+2 \overline{\alpha^{*}}\right), \\
g_{02} & =2 \bar{D} \tau_{i}\left(\bar{\alpha}+\overline{\alpha^{*}}\right), \\
g_{21}= & \bar{D} \tau_{i}\left[2 W_{11}^{(2)}(0)+W_{20}^{(2)}(0)+2 \alpha W_{11}^{(1)}(0)\right. \\
& \left.\quad+\bar{\alpha} W_{20}^{(1)}(0)+2 \overline{\alpha^{*}}\left(2 W_{11}^{(1)}(0)+W_{20}^{(1)}(0)\right)\right] .
\end{aligned}
$$

In order to assure the value of $g_{21}$, we need to compute $W_{20}(\theta)$ and $W_{11}(\theta)$. From (37) and (43), we have

$$
\begin{aligned}
\dot{W} & =\dot{u}_{t}-\dot{z} q-\dot{\bar{z}} \bar{q} \\
& = \begin{cases}A W-2 \operatorname{Re}\left\{\bar{q}^{*}(0) f_{0} q(\theta)\right\}, & \theta \in[0,1) \\
A W-2 \operatorname{Re}\left\{\bar{q}^{*}(0) f_{0} q(\theta)\right\}+f_{0}, & \theta=0\end{cases} \\
& \triangleq A W+H(z, \bar{z}, \theta),
\end{aligned}
$$

where

$$
H(z, \bar{z}, \theta)=H_{20}(\theta) \frac{z^{2}}{2}+H_{11}(\theta) z \bar{z}+H_{02}(\theta) \frac{\bar{z}^{2}}{2}+\cdots .
$$

Notice that, near the origin on the center manifold $C_{0}$, we have

$$
\dot{W}=W_{z} \dot{z}+W_{\bar{z}} \dot{\bar{z}} ;
$$

thus, we have

$$
\begin{gathered}
\left(A-2 i \omega_{k} \tau_{k} I\right) W_{20}(\theta)=-H_{20}(\theta), \\
A W_{11}(\theta)=-H_{11}(\theta) .
\end{gathered}
$$

Since (51), for $\theta \in[-1,0)$, we have

$$
\begin{aligned}
H(z, \bar{z}, \theta) & =-\overline{q^{*}}(0) f_{0} q(\theta)-q^{*}(0) \bar{f}_{0} \bar{q}(\theta) \\
& =-g q(\theta)-\bar{g} \bar{q}(\theta) .
\end{aligned}
$$

Comparing the coefficients with (51) gives that

$$
\begin{aligned}
& H_{20}(\theta)=-g_{20} q(\theta)-\bar{g}_{02} \bar{q}(\theta), \\
& H_{11}(\theta)=-g_{11} q(\theta)-\bar{g}_{11} \bar{q}(\theta) .
\end{aligned}
$$

From (54), (56), and the definition of $A$, we can get

$$
\dot{W}_{20}(\theta)=2 i \omega_{i} \tau_{i} W_{20}(\theta)+g_{20} q(\theta)+\bar{g}_{02} \bar{q}(\theta) .
$$

Notice that $q(\theta)=q(0) e^{i \omega_{i} \tau_{i} \theta}$, and we have

$$
W_{20}(\theta)=\frac{i g_{20}}{\omega_{i} \tau_{i}} q(0) e^{i \omega_{i} \tau_{i} \theta}+\frac{i \bar{g}_{02}}{3 \omega_{i} \tau_{i}} \bar{q}(0) e^{-i \omega_{i} \tau_{i} \theta}+E_{1} e^{2 i \omega_{i} \tau_{i} \theta},
$$

where $E_{1}=\left(E_{1}^{(1)}, E_{1}^{(2)}, E_{1}^{(3)}, E_{1}^{(4)}\right)^{T} \in \mathbb{R}^{4}$ is a constant vector. In the same way, we can also obtain

$$
W_{11}(\theta)=-\frac{i g_{11}}{\omega_{i} \tau_{i}} q(0) e^{i \omega_{i} \tau_{i} \theta}+\frac{i \bar{g}_{11}}{\omega_{i} \tau_{i}} \bar{q}(0) e^{-i \omega_{i} \tau_{i} \theta}+E_{2},
$$

where $E_{2}=\left(E_{2}^{(1)}, E_{2}^{(2)}, E_{2}^{(3)}, E_{2}^{(4)}\right)^{T} \in \mathbb{R}^{4}$ is also a constant vector.

In what follows, we will compute $E_{1}$ and $E_{2}$. From the definition of $A$ and (54), we have

$$
\begin{aligned}
& \int_{-1}^{0} d \eta(\theta) W_{20}(\theta)=2 i \omega_{i} \tau_{i} W_{20}(0)-H_{20}(0), \\
& \int_{-1}^{0} d \eta(\theta) W_{11}(\theta)=-H_{11}(0) .
\end{aligned}
$$


From (51) and (52), we have

$$
\begin{gathered}
H_{20}(0)=-g_{20} q(0)-\bar{g}_{02} \bar{q}(0)+2 \tau_{i}[\alpha, 1,0,0]^{T}, \\
H_{11}(0)=-g_{11} q(0)-\bar{g}_{11} \bar{q}(0)+2 \tau_{i}[\operatorname{Re}\{\alpha\}, 1,0,0]^{T} .
\end{gathered}
$$

Substituting (58) and (62) into (60) and noticing that

$$
\begin{gathered}
{\left[i \omega_{i} \tau_{i} I-\int_{-1}^{0} e^{i \omega_{i} \tau_{i} \theta} d \eta(\theta)\right] q(0)=0} \\
{\left[-i \omega_{i} \tau_{i} I-\int_{-1}^{0} e^{-i \omega_{i} \tau_{i} \theta} d \eta(\theta)\right] \bar{q}(0)=0,}
\end{gathered}
$$

we obtain

$$
\begin{aligned}
& {\left[\begin{array}{cccc}
2 i \omega_{i}+a-y^{*} & -x^{*} & -1 & 0 \\
-2 x^{*} & 2 i \omega_{i}+b & 0 & 0 \\
1 & 0 & 2 i \omega_{i}+c & -1 \\
0 & 0 & -k+k e^{-i \omega_{i} \tau_{i}} & 2 i \omega_{i}+d
\end{array}\right] E_{1}} \\
& \quad=2 \tau_{i}\left[\begin{array}{l}
\alpha \\
1 \\
0 \\
0
\end{array}\right]
\end{aligned}
$$

That is,

$$
\begin{aligned}
E_{1}= & 2 \tau_{i}\left[\begin{array}{cccc}
2 i \omega_{i}+a-y^{*} & -x^{*} & -1 & 0 \\
-2 x^{*} & 2 i \omega_{i}+b & 0 & 0 \\
1 & 0 & 2 i \omega_{i}+c & -1 \\
0 & 0 & -k+k e^{-i \omega_{i} \tau_{i}} & 2 i \omega_{i}+d
\end{array}\right]^{-1} \\
& \times\left[\begin{array}{c}
\alpha \\
1 \\
0 \\
0
\end{array}\right] .
\end{aligned}
$$

Similarly, substituting (59) and (63) into (61), we can get the formula of $E_{2}$, where

$$
E_{2}=2 \tau_{i}\left[\begin{array}{cccc}
a-y^{*} & -x^{*} & -1 & 0 \\
-2 x^{*} & b & 0 & 0 \\
1 & 0 & c & -1 \\
0 & 0 & -k & d
\end{array}\right]^{-1}\left[\begin{array}{c}
\operatorname{Re}\{\alpha\} \\
1 \\
0 \\
0
\end{array}\right]
$$

Thus, we can determine $W_{20}(\theta)$ and $W_{11}(\theta)$. Furthermore, we can determine each $g_{i j}$. Therefore, each $g_{i j}$ is determined by the parameters and delay in (4). Thus, we can compute the following values:

$$
\begin{aligned}
c_{1}(0) & =\frac{i}{2 \omega_{i} \tau_{i}}\left(g_{20} g_{11}-2\left|g_{11}\right|^{2}-\frac{1}{3}\left|g_{02}\right|^{2}\right)+\frac{1}{2} g_{21}, \\
\mu_{2} & =-\frac{\operatorname{Re}\left\{c_{1}(0)\right\}}{\operatorname{Re}\left\{\lambda^{\prime}(0)\right\}}, \\
T_{2} & =-\frac{\operatorname{Im}\left\{c_{1}(0)\right\}+\mu_{2} \operatorname{Im}\left\{\lambda^{\prime}(0)\right\}}{\omega_{i} \tau_{i}}, \\
\beta_{2} & =2 \operatorname{Re}\left\{c_{1}(0)\right\},
\end{aligned}
$$

which determine the quantities of bifurcating periodic solutions in the center manifold at the critical value $\tau_{i}$; that is, $\mu_{2}$ determines the directions of the Hopf bifurcation; if $\mu_{2}>0$ $(<0)$, then the Hopf bifurcation is supercritical (subcritical) and the bifurcation exists for $\tau>\tau_{i}\left(<\tau_{i}\right) ; \beta_{2}$ determines the stability of the bifurcation periodic solutions; the bifurcating periodic solutions are stable (unstable) if $\beta_{2}<0(>0)$; and $T_{2}$ determines the period of the bifurcating periodic solutions: the period increases (decreases) if $T_{2}>0(<0)$.

\section{Numerical Simulation}

In this section, we present some numerical results to verify the analytical predictions obtained in the previous section. These numerical simulation results constitute excellent validations of our theoretical analysis; it is shown that the chaotic orbit can be controlled to a periodic orbit by using washoutfilter-aided controller with time delay.

Figure 1 shows that system (1) is chaotic when $a=4, b=$ 0.1 , and $c=1$.

5.1. Hopf Bifurcation from the Stable Equilibrium $P_{0}$. In this subsection, we choose $a=4, b=0.4$, and $c=1$; then, system (1) has only a stable equilibrium $P_{0}(0,2.5,0)$. From the algorithm of Section 3, we get that $r=(1.5 d-1.5 k)^{2}-$ $2.25 k^{2}$; thus, if $k>d / 2$, then $r<0$. From Lemma 4 , (15) has at least one positive root. Let $d=1$ and $k=1$. From the algorithm of Section 3, we can compute $\tau_{0} \doteq$ 5.5932. Thus, from Theorem 5 , the equilibrium $P_{0}(0,2.5,0)$ is asymptotically stable when $\tau<\tau_{0}$, and, as $\tau$ crosses $\tau_{0}$, there are periodic orbits bifurcating from $P_{0}(0,2.5,0)$ (Figure 2 ).

5.2. Hopf Bifurcation from the Stable Equilibria $P_{ \pm}$. In this subsection, we choose $a=4, b=0.1$, and $c=4$; then, from Lemma 3, system (1) has three equilibria: $P_{0}(0,10,0)$ is unstable and $P_{ \pm}( \pm 0.7416,4.5, \mp 0.3708)$ are stable (Figure 3$)$. If we choose $k=2$, and $d=1$, from the algorithm of Section 3, we can get that the bifurcating value of $\tau$ is $\tau_{h}=2.3542$. When $\tau$ pass through $\tau_{h}=2.3542$, a family of periodic orbits will bifurcate from equilibria $P_{ \pm}$, respectively (Figure 4).

5.3. Application to Control of Chaos. From Figure 1, we can see that system (1) is chaotic when $a=4, b=0.1$, and $c=1$. If we choose $k=d=1$, a family of periodic orbits bifurcate from the equilibria of system (4) at some critical values of $\tau$. This can be verified by Figure 5 .

\section{Conclusion}

In this paper, we have investigated a financial system with time-delayed washout-filters-aided controller. Taking the time delay $\tau$ as bifurcating parameter, we discussed the conditions at which periodic orbits bifurcate from the equilibria $P_{0}$ and $P_{ \pm}$, respectively. The stability and direction of bifurcated periodic solutions have been also investigated in detail. And the obtained results can be applied to control the chaos of this financial system. 

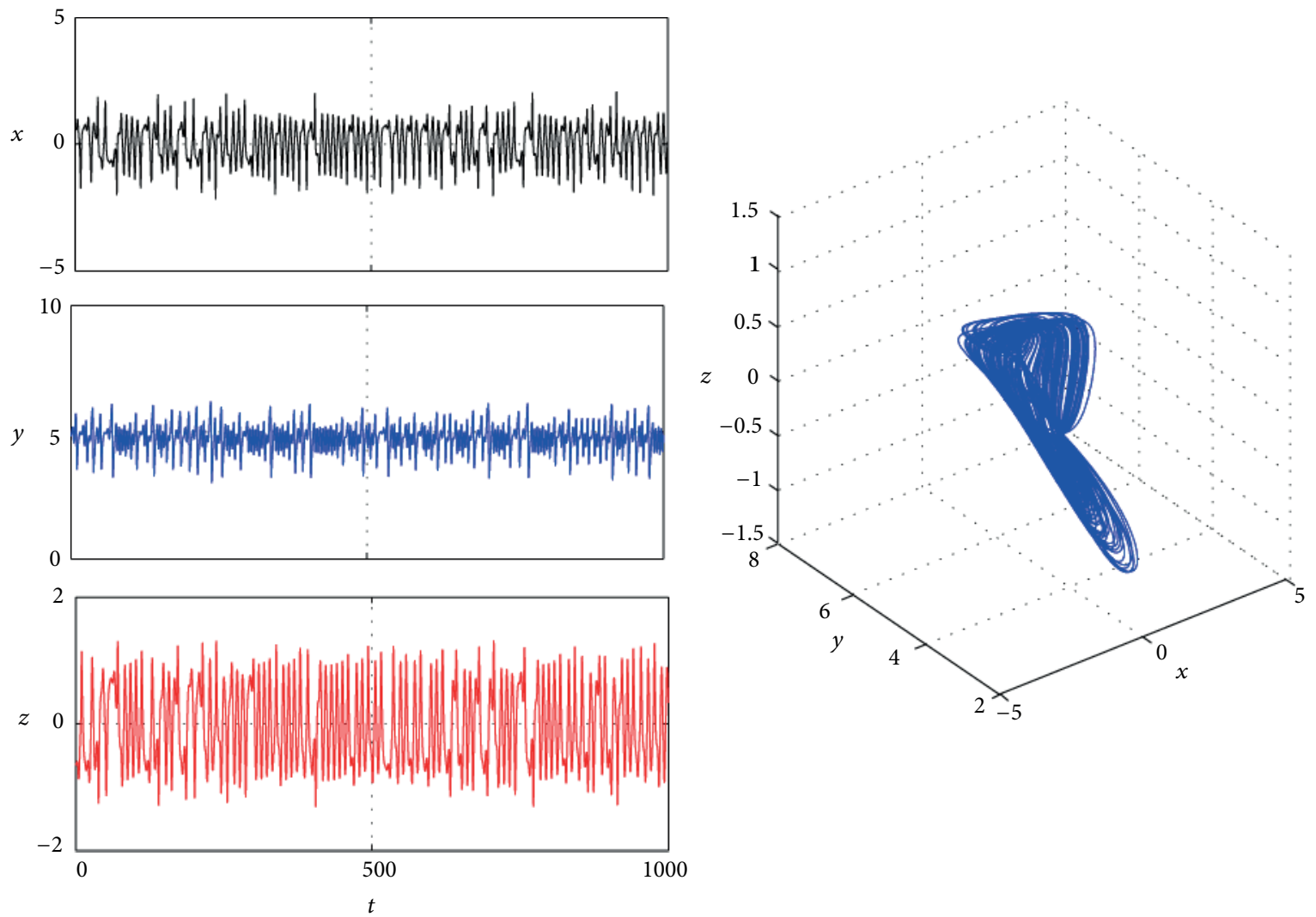

FIGURE 1: Trajectories $x(t), y(t)$, and $z(t)$ and phase graphs of system (1) with $a=4, b=0.1$, and $c=1$.
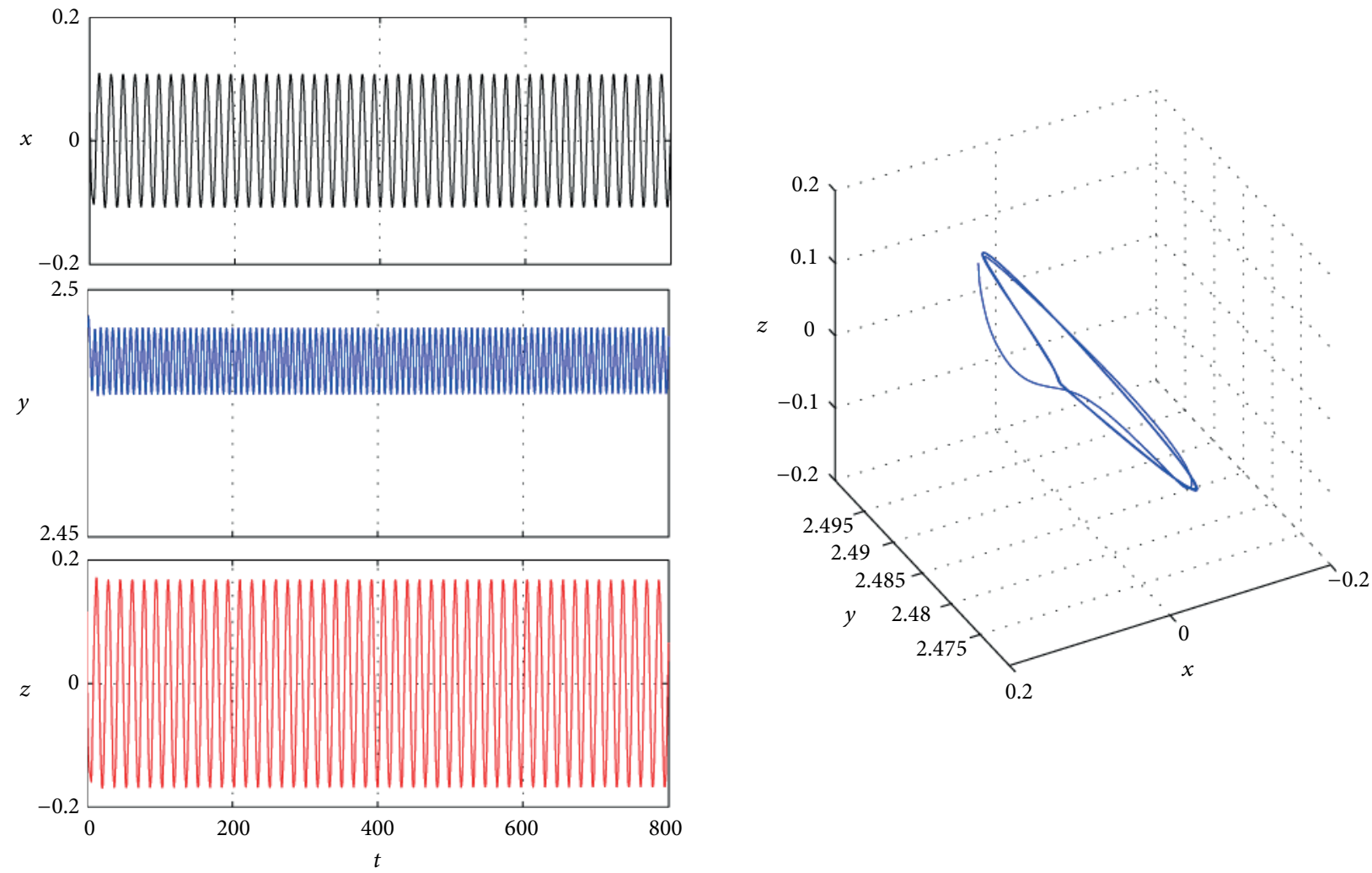

FIGURE 2: Trajectories $x(t), y(t)$, and $z(t)$ and phase graphs of system (4) with $a=4, b=0.4, c=1, k=1, d=1$, and $\tau=5.5940$. 

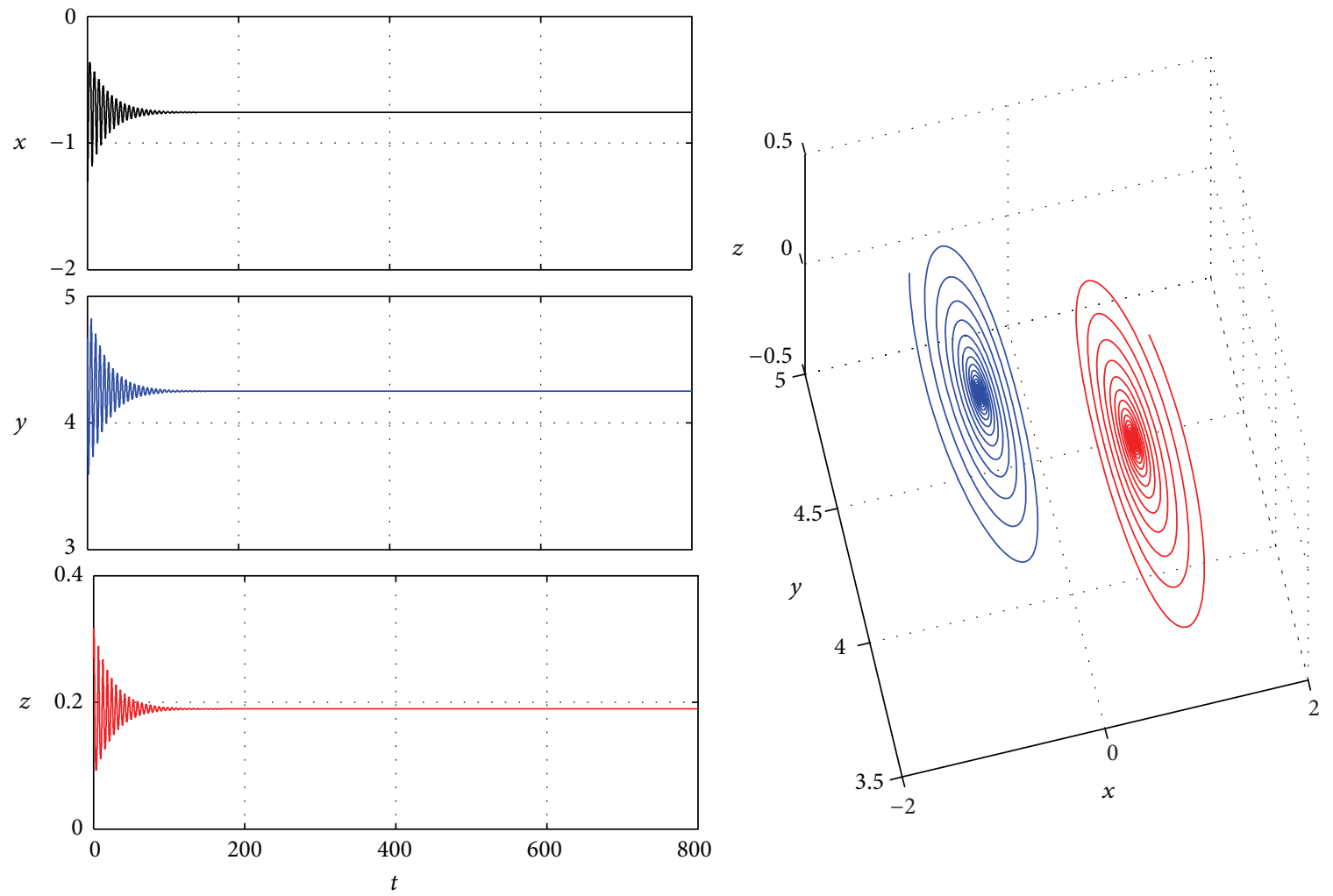

FIgUre 3: Trajectories $x(t), y(t)$, and $z(t)$ and phase graphs of system (1) with $a=4, b=0.1$, and $c=4$.
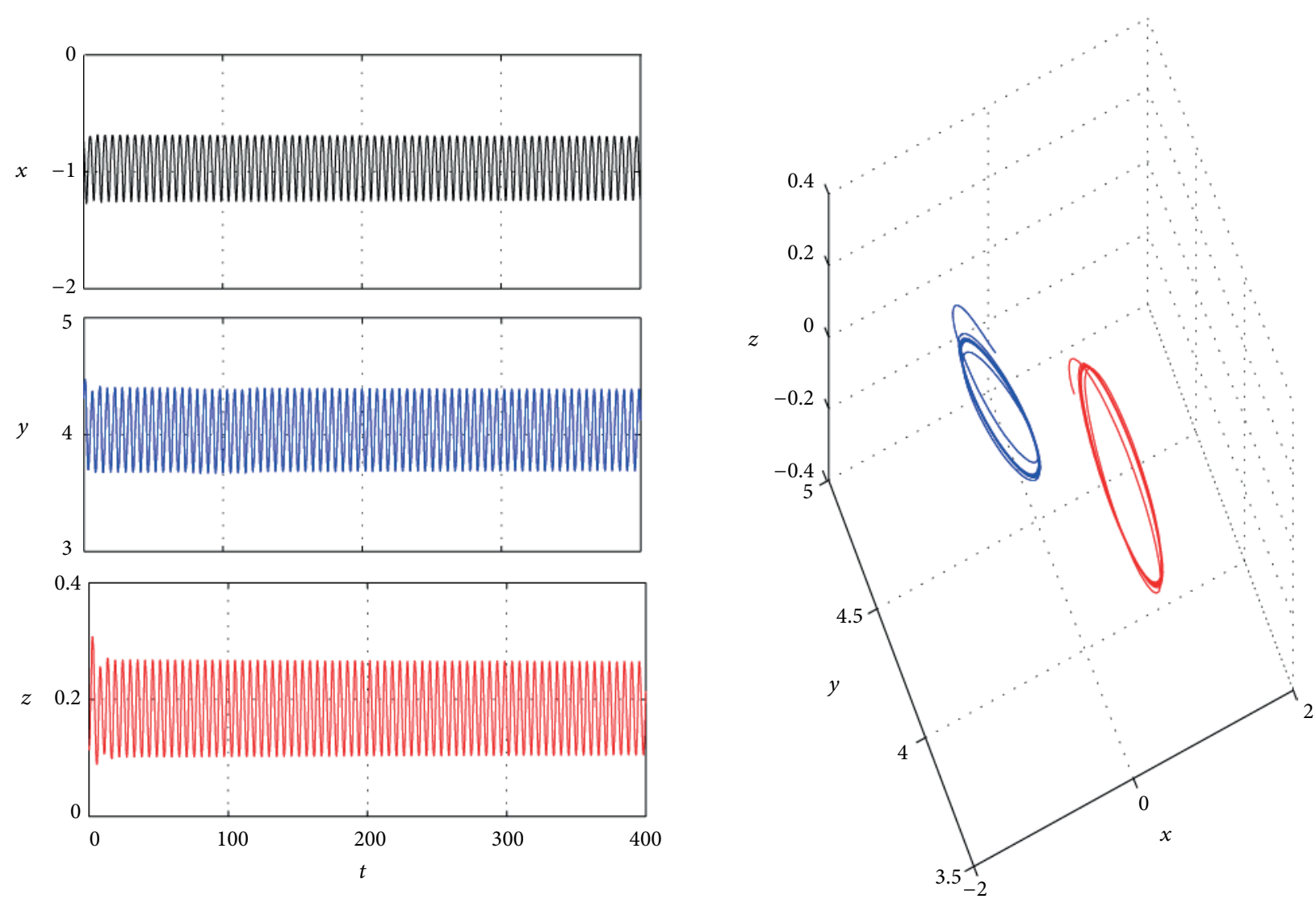

Figure 4: Trajectories $x(t), y(t)$, and $z(t)$ and phase graphs of system (4) with $a=4, b=0.1, c=4, k=2, d=1$, and $\tau=2.37$. 

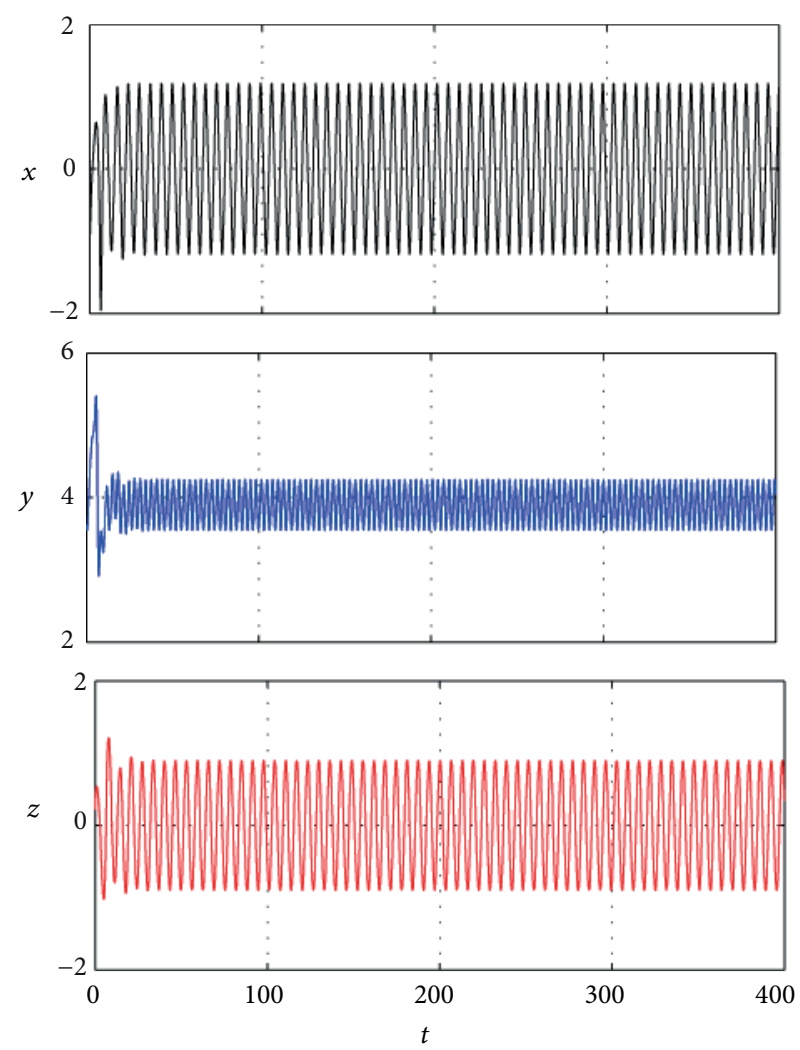

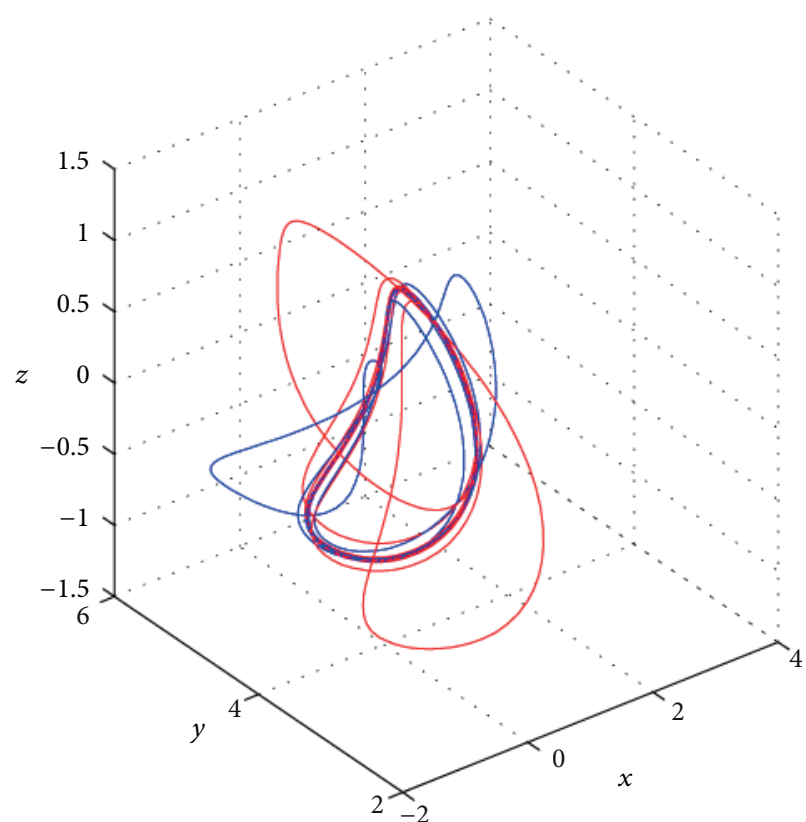

Figure 5: Trajectories $x(t), y(t)$, and $z(t)$ and phase graphs of system (4) with $a=4, b=0.1, c=1, k=1, d=1$, and $\tau=2$.
From a financial sense, the obtained results show that the delay on price index has great influence on the financial system, which can be applied to suppress or avoid the chaos phenomenon appearing in the financial system, so as to make the economic system run well. On the other hand, the control gain $k$ is also applied to influence the dynamical behaviors of this financial system; it will be investigated in the near future.

\section{Conflict of Interests}

The authors declare that there is no conflict of interests regarding the publication of this paper.

\section{Acknowledgments}

The authors would like to thank the anonymous referee for the very helpful suggestions and comments which led to the improvement of the original paper. And this work is supported by Science and Technology Department of Henan Province (122300410417), Education Department of Henan Province (13A110108), 2013 Scientific Research Project of Beifang University of Nationalities (2013XYZ021), Institute of Information and System Computation Science of Beifang University (13xyb01).

\section{References}

[1] R. M. Goodwin, Chaotic Economic Dynamics, Cambridge University Press, Oxford, UK, 1990.

[2] R. Shone, Economic Dynamics, Cambridge University Press, Cambridge, UK, 2002.

[3] R. M. Goodwin, "The nonlinear accelerator and the persistence of business cycles," Econometrica, vol. 19, no. 1, pp. 1-17, 1951.

[4] A. C.-L. Chian, E. L. Rempel, and C. Rogers, "Complex economic dynamics: chaotic saddle, crisis and intermittency," Chaos, Solitons and Fractals, vol. 29, no. 5, pp. 1194-1218, 2006.

[5] L. Fanti and P. Manfredi, "Chaotic business cycles and fiscal policy: an IS-LM model with distributed tax collection lags," Chaos, Solitons and Fractals, vol. 32, no. 2, pp. 736-744, 2007.

[6] J. Ma and Y. Chen, "Study for the bifurcation topological structure and the global complicated character of a kind of nonlinear finance system (I)," Applied Mathematics and Mechanics, vol. 22, no. 11, pp. 1119-1128, 2001.

[7] J. Ma and Y. Chen, "Study for the bifurcation topological structure and the global complicated character of a kind of nonlinear finance system (II)," Applied Mathematics and Mechanics, vol. 22, no. 12, pp. 1236-1242, 2001.

[8] C. Ma and X. Wang, "Hopf bifurcation and topological horseshoe of a novel finance chaotic system," Communications in Nonlinear Science and Numerical Simulation, vol. 17, no. 2, pp. 721-730, 2012.

[9] Q. Gao and J. Ma, "Chaos and Hopf bifurcation of a finance system,” Nonlinear Dynamics, vol. 58, no. 1-2, pp. 209-216, 2009. 
[10] W.-C. Chen, "Dynamics and control of a financial system with time-delayed feedbacks," Chaos, Solitons and Fractals, vol. 37, no. 4, pp. 1198-1207, 2008.

[11] R. G. D. Allen, Mathematical Economics, Macmillan, London, UK, 1963.

[12] J. B. Taylor, Macroeconomic Policy in a World Economy, WW Norton, New York, NY, USA, 1999.

[13] J. K. Hale and S. M. Lunel, Introduction to Functional Differential Equations, Springer, New York, NY, USA, 1993.

[14] Z. Cheng, "Anti-control of Hopf bifurcation for Chen's system through washout filters," Neurocomputing, vol. 73, no. 16-18, pp. 3139-3146, 2010.

[15] W. Du, Y. Chu, J. Zhang, Y. Chang, J. Yu, and X. An, "Control of Hopf bifurcation in autonomous system based on washout filter," Journal of Applied Mathematics, vol. 2013, Article ID 482351, 16 pages, 2013. 


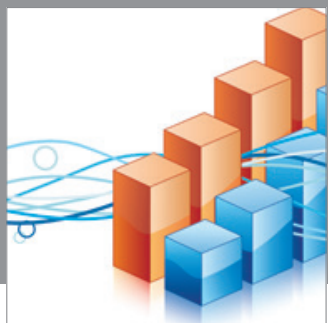

Advances in

Operations Research

mansans

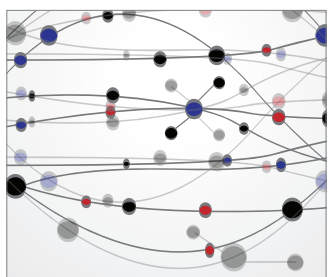

The Scientific World Journal
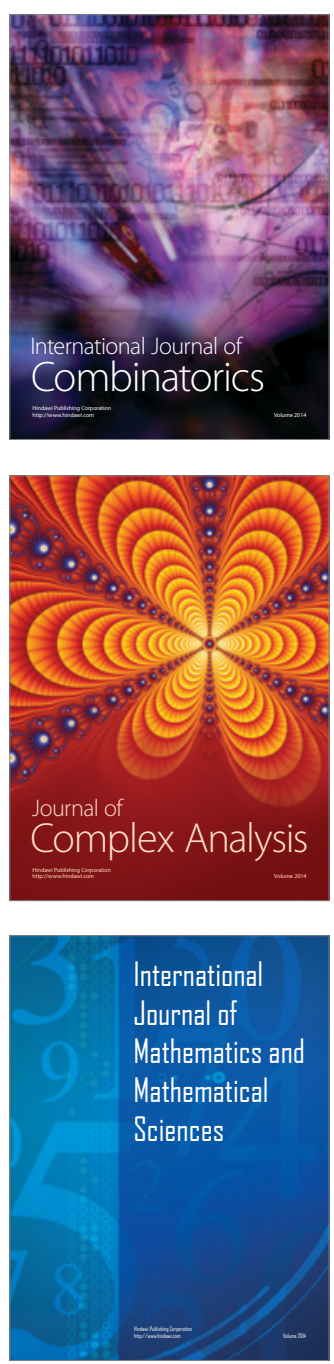
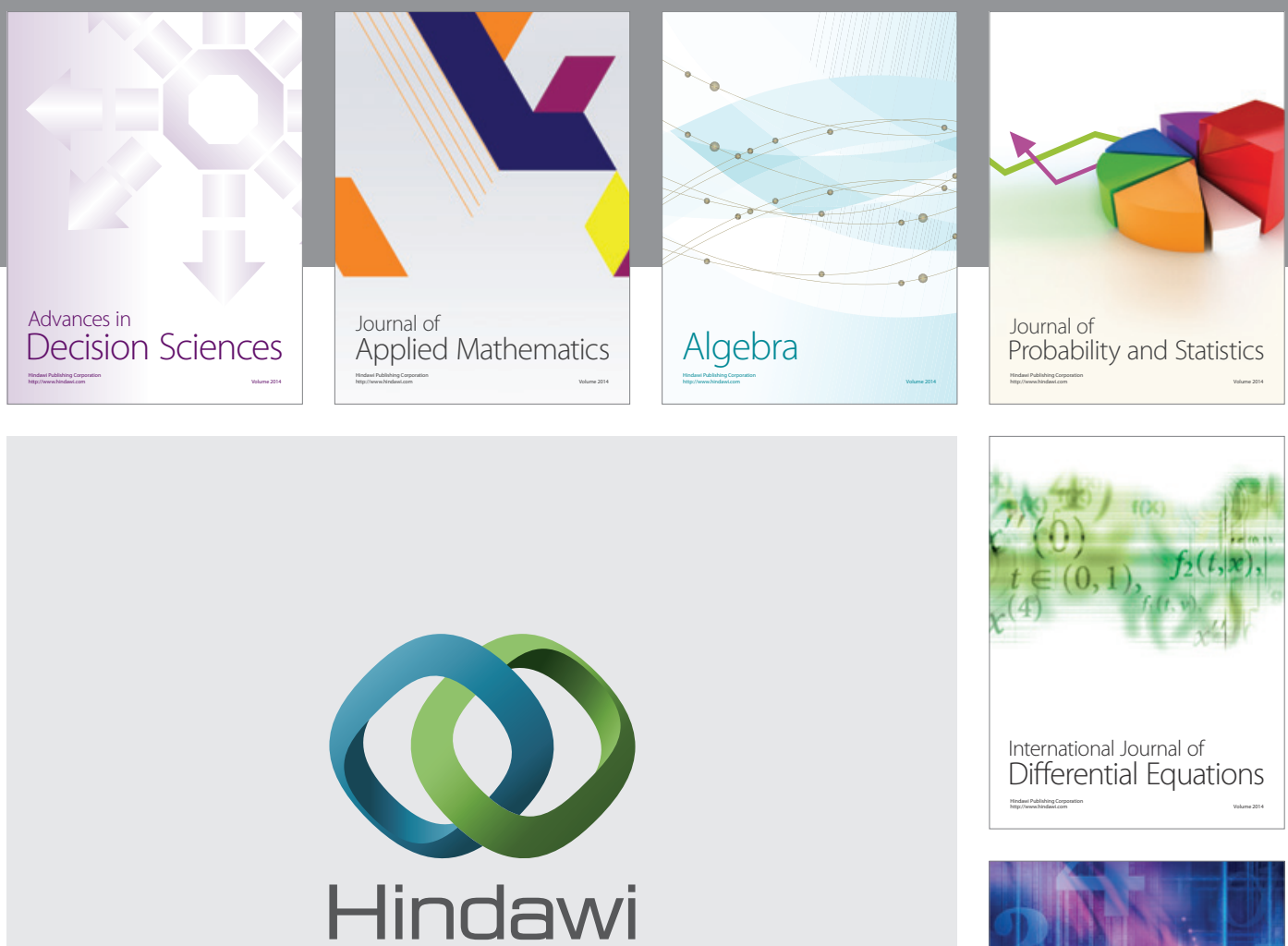

Submit your manuscripts at http://www.hindawi.com
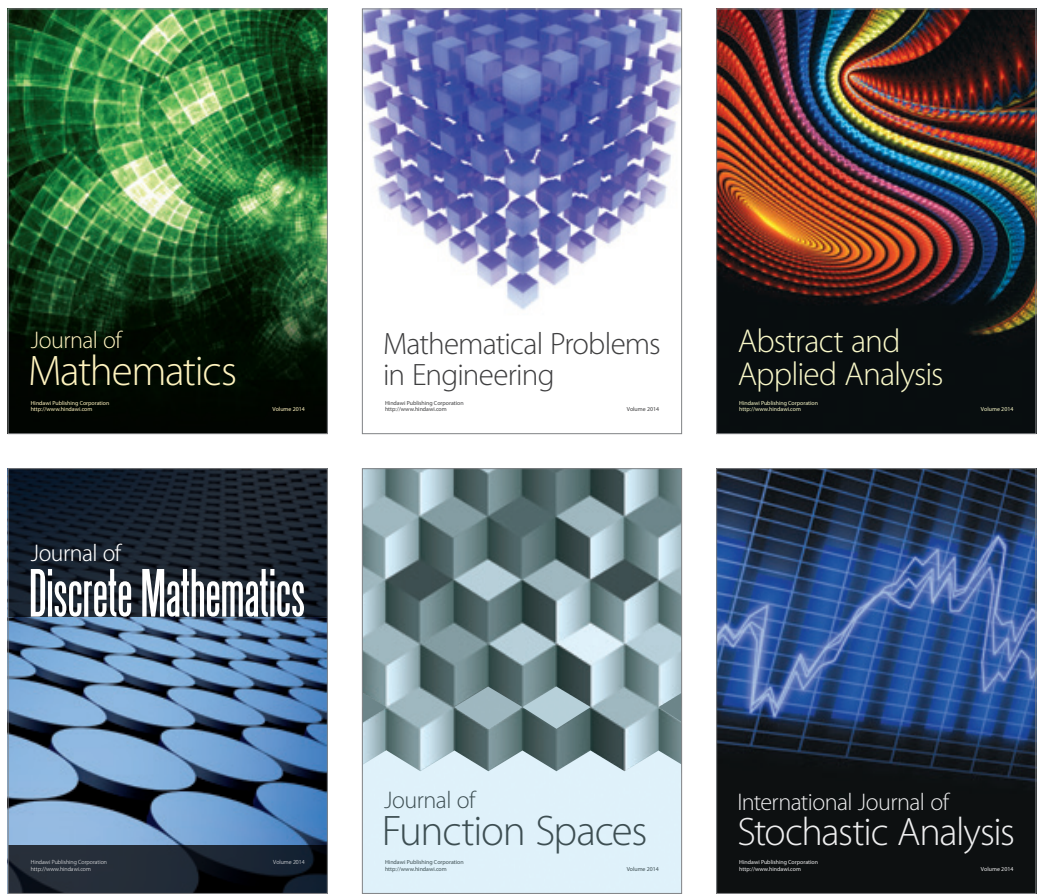

Journal of

Function Spaces

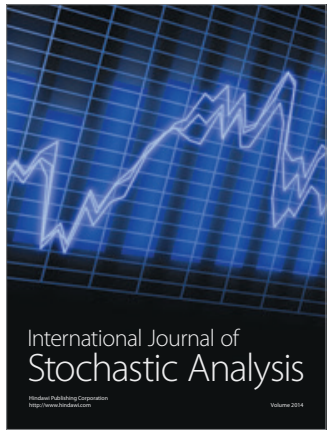

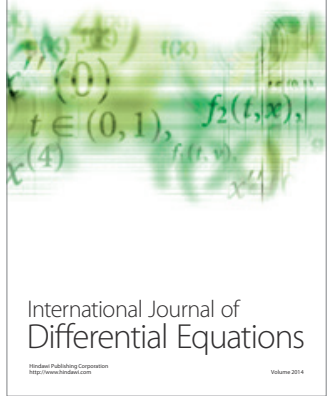
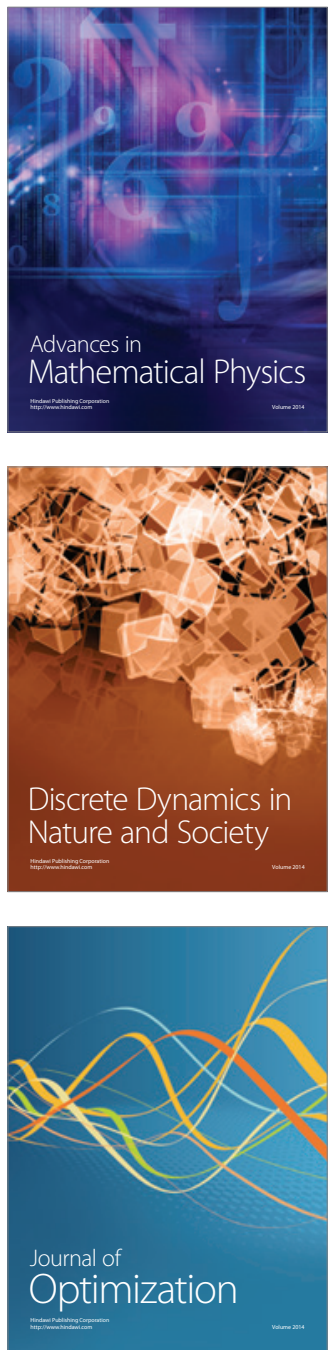